\title{
Current-voltage relationship in the auroral particle acceleration region
}

\author{
M. Morooka ${ }^{1,{ }^{*}}$, T. Mukai ${ }^{1}$, and H. Fukunishi ${ }^{2}$ \\ ${ }^{1}$ Institute of Space and Astronautical Science, Sagamihara, Kanagawa, Japan \\ ${ }^{2}$ University Tohoku, Sendai, Miyagi, Japan \\ * present address: Swedish Institute of Space Physics, Uppsala, Sweden
}

Received: 23 February 2004 - Revised: 19 July 2004 - Accepted: 17 August 2004 - Published: 3 November 2004

\begin{abstract}
The current-voltage relationship in the auroral particle acceleration region has been studied statistically by the Akebono (EXOS-D) satellite in terms of the charge carriers of the upward field-aligned current. The Akebono satellite often observed field-aligned currents which were significantly larger than the model value predicted by Knight (1973). We compared the upward field-aligned current estimated by three different methods, and found that low-energy electrons often play an important role as additional current carriers, together with the high-energy primary electrons which are expected from Knight's relation. Such additional currents have been observed especially at high and middle altitudes of the particle acceleration region. Some particular features of electron distribution functions, such as "cylindrical distribution functions" and "electron conics", have often been observed coinciding with the additional currents. They indicated time variability of the particle acceleration region. Therefore, we have concluded that the low-energy electrons within the "forbidden" region of electron phase space in the stationary model often contribute to charge carriers of the current because of the rapid time variability of the particle acceleration region. "Cylindrical distribution functions" are expected to be found below the time-varying potential difference. We statistically examined the locations of "cylindrical distribution function", and found that their altitudes are related to the location where the additional currents have been observed. This result is consistent with the idea that the lowenergy electrons can also carry significant current when the acceleration region changes in time.
\end{abstract}

Key words. Magnetospheric physics (auroral phenomena; current systems; magnetosphere-ionosphere interactions)

\section{Introduction}

The field-aligned currents play a key role in the magnetosphere-ionosphere coupling system. In particular,

Correspondence to: M. Morooka

(morooka@irfu.se) the upward field-aligned currents are associated with the inverted-V auroral particle acceleration events (e.g. Frank and Ackerson, 1971; Bryant et al., 1978; Kaya et al., 1981) and therefore, they are believed to relate closely to generation mechanisms of the parallel electric field. Various mechanisms for the generation of parallel electric fields have been discussed for a few decades (Borovsky, 1993, and reference therein). A relationship between the field-aligned current density $\left(J_{\|}\right)$and the potential difference $\left(V_{\|}\right)$has been predicted by Knight (1973).

$J_{\|}=e N_{S} \frac{B}{B_{E}} \sqrt{\frac{k T_{S}}{2 \pi m_{e}}}\left(1+\frac{e V_{\|}}{k T_{S}}\right)$,

where $B$ and $B_{E}$ are the magnetic strengths at an observation point and the ionosphere, $m_{e}$ is the electron mass, and $N_{S}$ and $k T_{S}$ are the number density and thermal energy of the magnetospheric electrons, respectively. In the limit of $e V_{\|} \ll k T_{S}$, the Eq. 1 becomes approximately linear. This model relies upon several assumptions:

- The charge carriers of the currents are only the magnetospheric electrons with an isotropic Maxwellian distribution function in the source region.

- A time-stationary parallel electric field.

- The energy and the magnetic moment are conserved along the magnetic field line.

This relationship has been studied theoretically (Chiu and Schulz, 1978; Lyons, 1980; Gurgiolo and Burch, 1988; Rönnmark, 2002) and observationally, as summarized in Table 1. However, the validity of Knight's relation is still controversial. Among the six papers which examined the current-voltage relationship quantitatively (marked papers in Table 1), three papers reported good agreements of the relationship. Note that most of these results are obtained at low altitudes below the auroral particle acceleration region. On the other hand, Sakanoi et al. (1995) have shown that the observed field-aligned currents are about 2-20 times larger than the model currents using data from the Akebono satellite at 
Table 1. Summary of previous studies about the current-voltage relationship in the upward field-aligned current region. The marked papers have examined $(*)$ the Knight's current-voltage relationship directly using particle data. Underlined papers have reported agreement with Knight's relation.

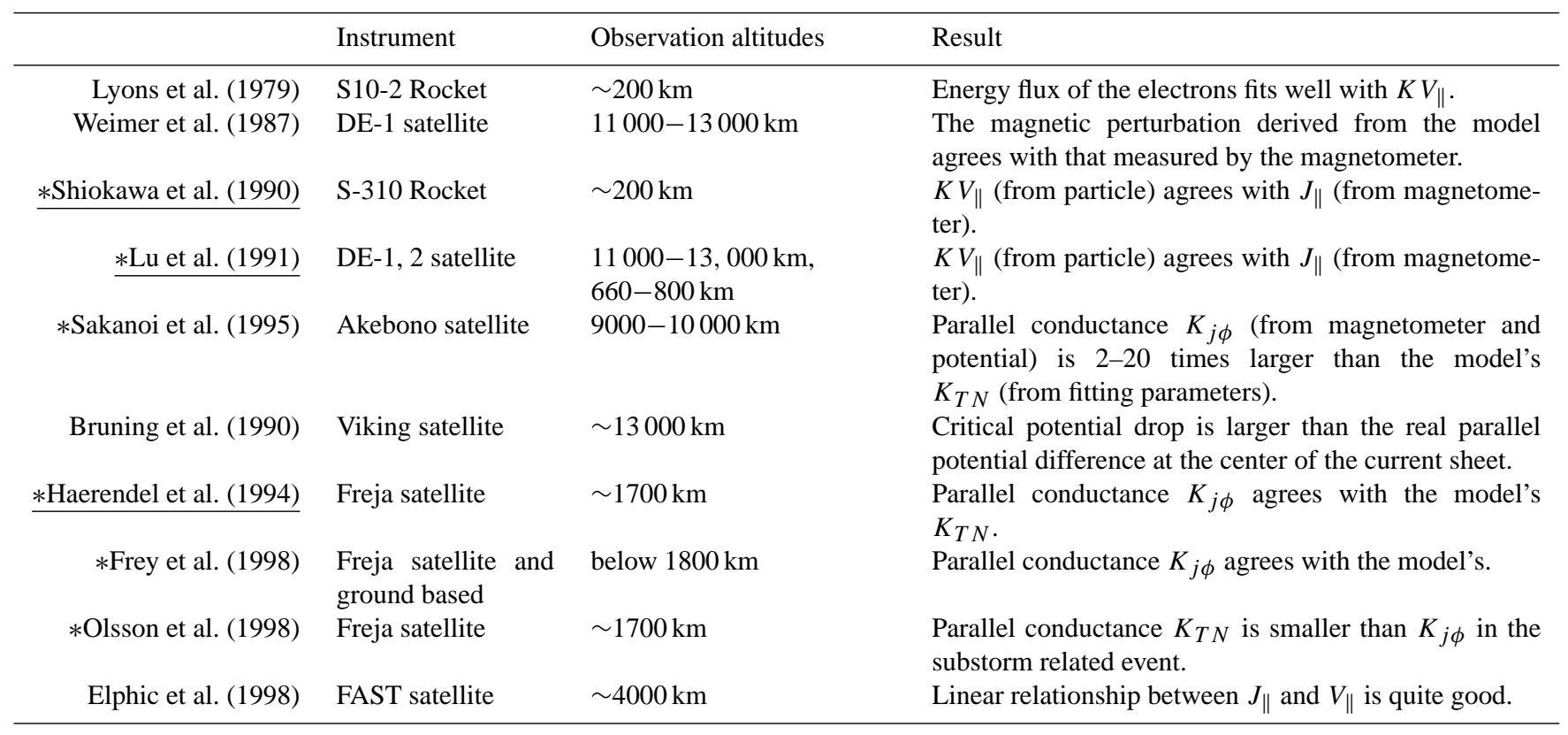

altitudes of 9000-10000 km. The parallel electric fields generally exist at altitudes of $3000-15000 \mathrm{~km}$ (e.g. Reiff et al., 1993). Hence, Akebono was located in the middle of the acceleration region, while the other results are obtained by the satellites located well above or below the particle acceleration region. The discrepancy between these results from the previous studies may be due to the difference of the observation altitudes. A large amount of data taken during the eight years of observation by Akebono, which can cover the altitudes of $3000-10000 \mathrm{~km}$ over the auroral region in the nightside and dayside, makes it possible to investigate the altitudinal dependence of auroral particle acceleration region.

The purpose of this study is to examine the current-voltage relationship in the acceleration region statistically, using data obtained by Akebono, and discuss the results in terms of charge carriers of the upward field-aligned currents. With the advantage of using a wide-range of coverage of altitudes up to $\sim 10000 \mathrm{~km}$, we have investigated latitudinal, local time, altitudinal, and seasonal characteristics of the current-voltage relationship. We will show that the field-aligned current densities often exceed Knight's model current, and that low energy electrons play an important role as additional current carriers in this case. By examining the distribution function of electrons in detail, we suggest that the low-energy electrons within the "forbidden" region of electron phase space in the static particle acceleration model often contribute to the current when the particle acceleration region varies in time.

\section{Data analysis}

The Akebono (EXOS-D) satellite was launched on 21 February 1989 , into a semi-polar orbit with the initial perigee and apogee of $274 \mathrm{~km}$ and $10500 \mathrm{~km}$, respectively, to study mechanisms of the auroral particle acceleration by direct observations of particles and fields in the acceleration region. We examined the current-voltage relationship in the acceleration region, using LEP (the low energy particle detector, Mukai et al., 1990) and MGF (the magnetometer, Fukunishi et al., 1990) data obtained by Akebono. The LEP data provide three-dimensional distribution functions of electrons and positive ions with a time resolution of $\sim 8 \mathrm{~s}$. For magnetic field data, the 8-s (spin period) averaged fluxgate magnetometer data are used for the present analysis. In order to analyse only the auroral particle acceleration events, all data used for the statistical analysis in this study are the data which are regarded as an auroral electron acceleration event (where the accelerated Maxwellian distribution function has been detected) in Morooka and Mukai (2003). We have used only data which sufficiently cover the downward pitch angles $\left(0-30^{\circ}\right.$ in the Northern Hemisphere, and $150-180^{\circ}$ in the Southern Hemisphere) of electrons and the upward pitch angles $\left(150-180^{\circ}\right.$ in the Northern Hemisphere, and $0-30^{\circ}$ in the Southern Hemisphere) of ions (see Sect. 2 in Morooka and Mukai (2003) for details). Figure 1 shows data numbers of events used in the statistical analysis. Each map consists of bins of $64^{\circ}-76^{\circ}$ in invariant latitudes and 24 magnetic local times, being divided into three altitude ranges of $3000-6000 \mathrm{~km}, 6000-8000 \mathrm{~km}$, and $8000-10000 \mathrm{~km}$. 
There are several methods to estimate the field-aligned currents in the auroral particle acceleration region. We calculate the field-aligned current densities with three different approaches.

\subsection{Current estimation using Knight's model: $J_{\text {model }}$}

The field-aligned currents $J_{\text {model }}$ are calculated using the model Eq. (1). The parameters ( $e \phi, N_{s}$, and $\left.T_{s}\right)$ in Eq. (1) are estimated from the distribution functions of electrons and ions as follows.

The potential difference $(\phi)$ is calculated as the sum of the potential differences above and below the observation point. The potential differences are determined by the peak energies of the distribution functions of downward electrons and upward ions, respectively $\left(\phi_{a}\right.$ and $\left.\phi_{b}^{\mathrm{UFI}}\right)$. When a potential difference exists above (or below) the satellite altitude, the magnetospheric electrons (or ionospheric ions) gain energies from the field-aligned electric field, and the accelerated Maxwellian distributions will be observed. The accelerated Maxwellian distribution can be written

$f(E)=N_{s}\left(\frac{m}{2 \pi k T_{s}}\right)^{\frac{3}{2}} \exp \left(-\frac{E-e \phi}{k T_{s}}\right)$,

where $m$ is the electron or ion (proton) mass, and $N_{s}$ and $k T_{s}$ are the number density and the thermal energy, respectively, in the source region (the magnetosphere for electron and ionosphere for ion), and $\phi$ is the potential difference above (and below) the observation point. We have also estimated the potential difference below the observation point from the energy-dependent width of the electron loss cone $\left(\phi_{b}^{L C}\right)$. When a potential difference exists below the satellite altitude, the angular boundary of the electron loss cone $\theta_{L C}$ is given by Cladis and Sharp (1979),

$\theta_{L C}=\sin ^{-1}\left(\frac{B_{o}}{B_{i}} \frac{E_{o}-e \phi_{b}}{E_{o}}\right)^{\frac{1}{2}}$

Here, the subscripts $o$ and $i$ indicate the observation point and the ionosphere level, respectively. For the estimation of the electron loss cone, we have used the loss cone search technique used in Sakanoi et al. (1995).

The number density $\left(N_{S}\right)$ and the thermal energy $\left(k T_{S}\right)$ are estimated by fitting the observed distribution function above the peak energy (here after, discrived as $E_{\text {peak }}$ ) to the accelerated Maxwellian distribution function (Eq. (2)) by a leastsquares method.

Examples of the distribution functions of electrons and ions observed in the particle acceleration region are shown in Fig. 2. The fitted accelerated Maxwellian distribution is represented by solid curves. In order to identify unambiguously acceleration events, cases of $\frac{e \phi}{k T_{s}}<3$ were excluded. In addition, we have checked the detected peak energy by visual inspection. electron data number used in the analysis

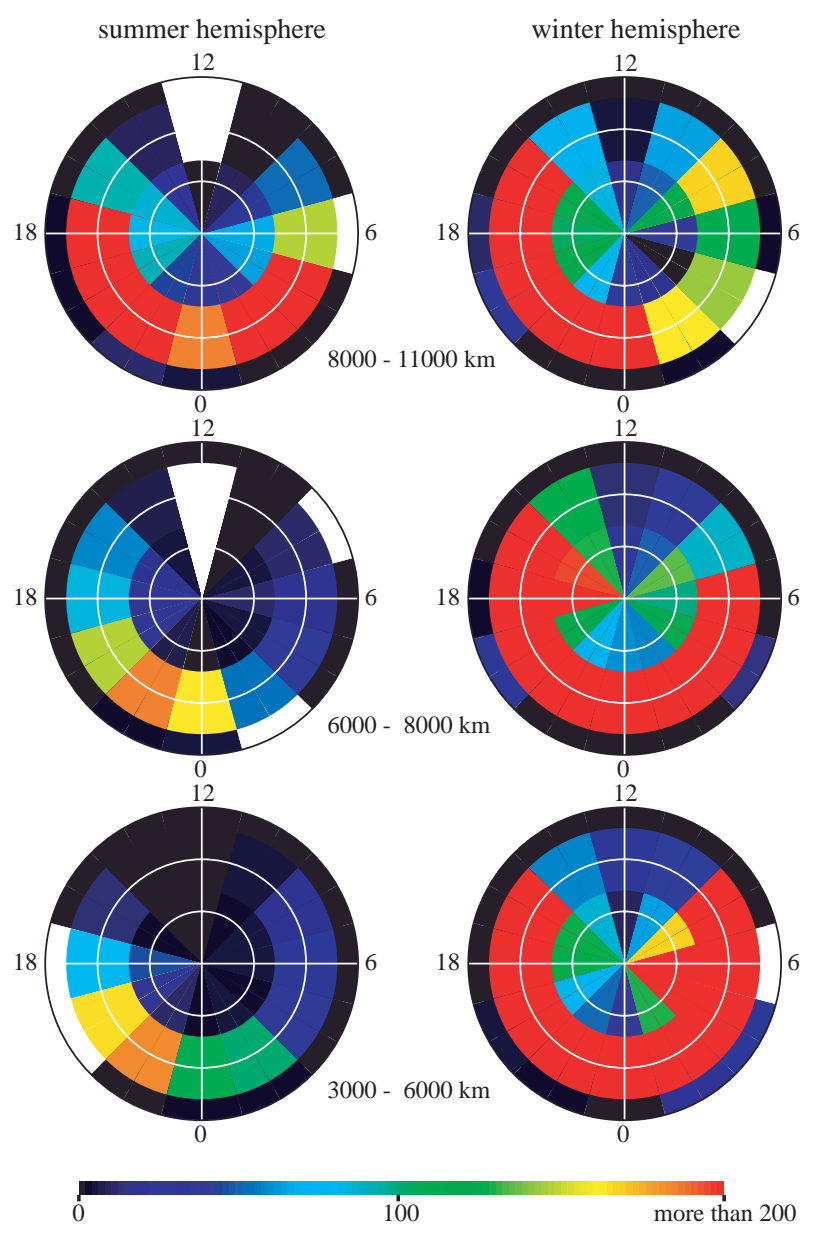

Fig. 1. The data number of accelerated electron events counted with units of the 8-s data point. The data are divided in MLT, MLAT, altitudes, and seasons. Each panel indicates the invariant latitude-local time map for each altitude range and season. The top panels indicate the high altitude range data above $8000 \mathrm{~km}$ to $10000 \mathrm{~km}$. The middle panels are for middle altitude range, $6000 \mathrm{~km}$ to $8000 \mathrm{~km}$. The bottom panels are for low altitude range below $6000 \mathrm{~km}$. The left and right side panels are data obtained in the summer and the winter hemisphere, respectively. The color bar is displayed in linear. The red color indicates the number of data more than 200

$$
\begin{aligned}
& \text { 2.2 Current estimation by integration of electrons: } \\
& J_{e^{-}}^{\text {low+high }}, J_{e^{-}}^{\text {high }}
\end{aligned}
$$

The field-aligned current densities can be estimated by integrating the particle differential flux $f$ over the whole energy and pitch angle range. The particle differential flux $F\left[/ \mathrm{cm}^{2} \mathrm{~s} \mathrm{str} \mathrm{eV}\right]$ is integrated by

$j_{\|}=-e \int_{E_{\min }}^{E_{\max }} d E \int_{0}^{\pi} 2 \pi F \sin (\theta) \cos (\theta) d \theta$,

where $e$ is the electron charge unit. Here, $E_{\min }$ is set at $100 \mathrm{eV}$ in order to eliminate spurious effects caused by 
EXOS-D LEP 90/20/06

$07: 35: 48-07: 35: 56$
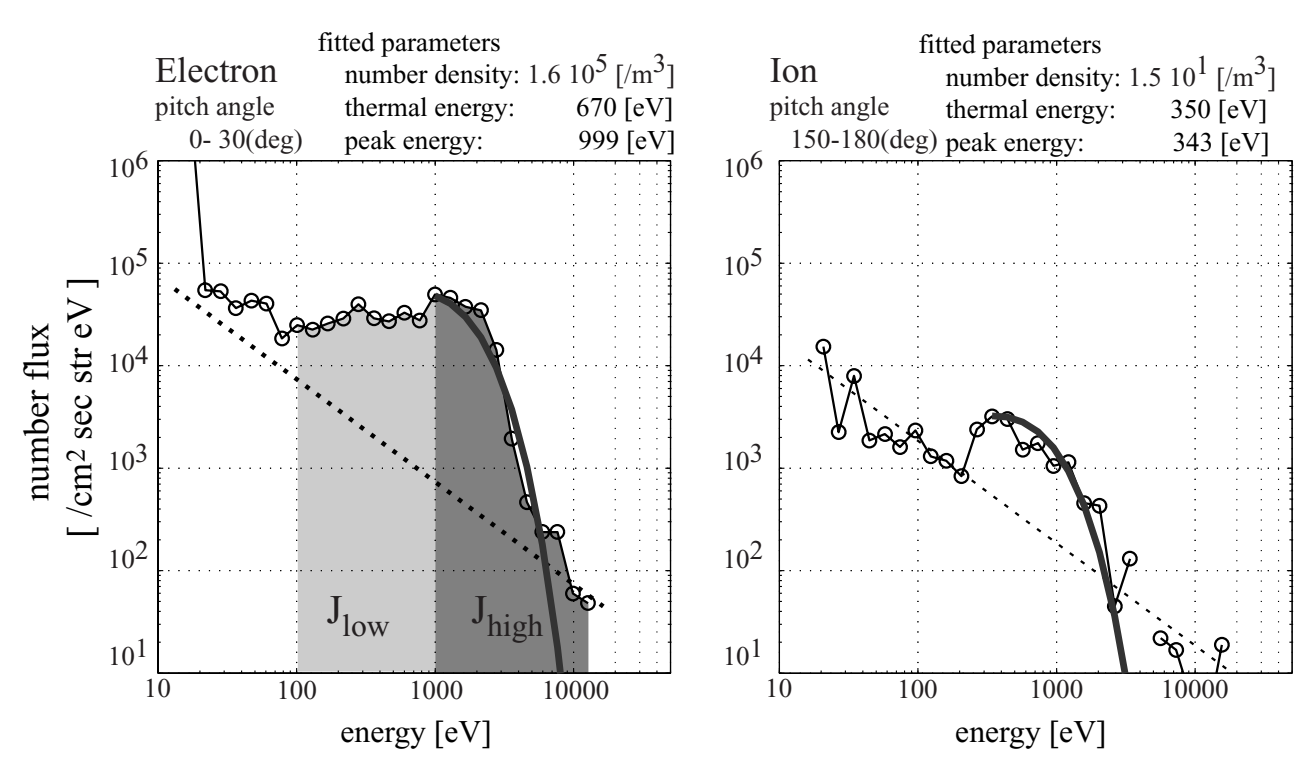

Fig. 2. Distribution functions of downward electrons and upward ions observed by Akebono/LEP in the particle acceleration region (represented in dot). Solid curves represent the fitted accelerated Maxwellian distribution. Fitted parameters are also shown in each panel. The distribution function above $E_{\text {peak }}$ (dark hatched area) was integrated to calculate $J_{e^{-}}^{\text {high }}$, and light hatched area was integrated to calculate $J_{\text {low }}$.

instrumental secondaries and so on. The value estimated by this method is written as $J_{e^{-}}^{\text {low+high }}$.

Knight (1973) assumed that the magnetospheric electrons, which are accelerated by the field-aligned potential difference, carry the field-aligned currents dominantly. The fieldaligned current densities carried by the magnetospheric electrons can be estimated by integrating the particle differential flux $f$ over all pitch angles above $E_{\text {peak }}$,

$j_{\|}=-e \int_{\text {peak energy }}^{E_{\max }} d E \int_{0}^{\pi} 2 \pi f \sin (\theta) \cos (\theta) d \theta$.

This value is described as $J_{e^{-}}^{\text {high }}$. It is expected that $J_{e^{-}}^{\text {low+high }}$ is almost equal to $J_{e^{-}}^{\text {high }}$, and also to Knight's model current $\left(J_{\text {model }}\right)$ in a time-stationary acceleration model.

\subsection{Current estimation by magnetic field data: $J_{B}^{\text {tot }}$}

The total field-aligned currents are estimated from the magnetometer data. With the assumption of a sheet structure, the field-aligned current density can be estimated from horizontal perturbations in the magnetic field, using Ampere's law,

$j_{\|}=-\frac{1}{\mu_{0}} \frac{\partial b}{\partial s}$,

where $\mu_{0}$ is the permeability in a vacuum, $b$ is the horizontal perturbation of the magnetic field, and $s$ is the normal distance from the current sheet. Discrete auroras are usually elongated along the east-west direction, hence the field-aligned current density is estimated from the east-west component of perturbation of the magnetic field in many previous studies. However, we have estimated the current densities more generally without assuming that the current sheets are aligned along the east-west direction. Assuming that $\boldsymbol{b}_{\mathbf{1}}$ and $\boldsymbol{b}_{\mathbf{2}}$ are the perturbation of the magnetic field at the locations corresponding to points $p_{1}$ and $p_{2}$, the spatial variation of the perturbed magnetic field is given by

$\Delta b=b_{2}-b_{1}$.

Note that $\Delta \boldsymbol{b}$ is aligned to the current sheet. The perpendicular width of the current sheet $\Delta \boldsymbol{w}$ is derived from the inner product of the normal vector of the current sheet $s$ and the spacecraft orbit vector $\boldsymbol{V}$, which is perpendicular to the ambient magnetic field component. Therefore, the field-aligned current density is given by

$j_{\|}=-\frac{1}{\mu_{0}} \frac{\partial b}{\partial w}$.

This value is defined as $J_{B}^{\text {tot }}$. When the angle between $\boldsymbol{s}$ and $\boldsymbol{V}$ is larger than $60^{\circ}$, the accuracy of the estimated current density is low. In this case, the obtained data are omitted.

By comparing $J_{B}^{\text {tot }}$ with $J_{e^{-}}^{\text {low+high }}$ and $J_{e^{-}}^{\text {high }}$, we can identify which particles dominantly contribute to the total current. In this study, $J_{e^{-}}^{\text {low }+ \text { high }}, J_{e^{-}}^{\text {high }}$, and $J_{B}^{\text {tot }}$ are mapped down to the ionospheric altitude $(120 \mathrm{~km})$, assuming a dipole field. 


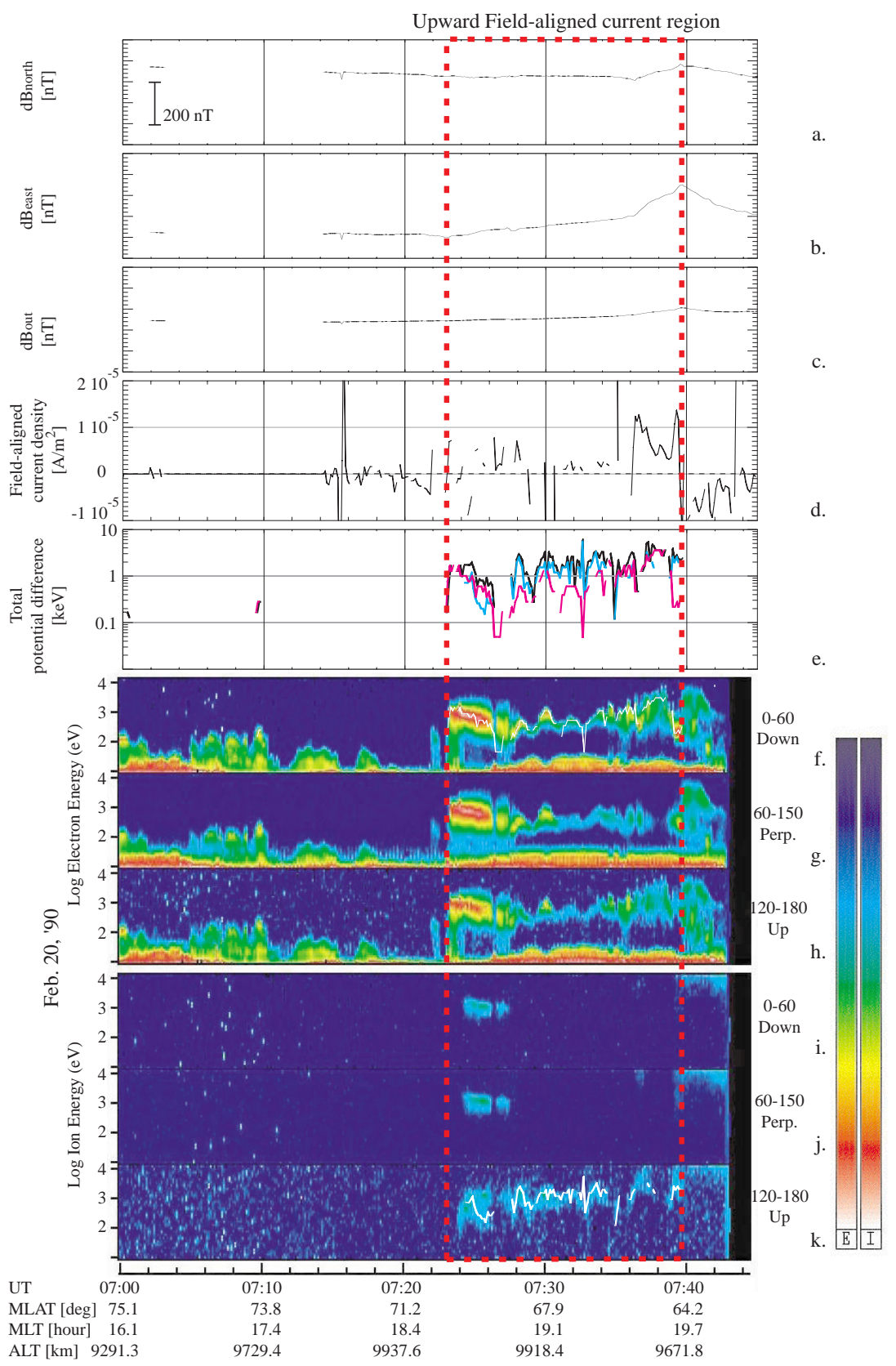

Fig. 3. Summary plot of the event 90022006 . (a-c) Magnetic field perturbation for the northward, eastward, and radial direction by MGF. (d) The field-aligned current density estimated by the magnetometer data. The estimated values are mapped down to the ionosphere (120 km) with assumption of the dipole magnetic field. (e) Potential differences estimated from the peak energies of the downward electrons and UFIs. Total (black), above (red), and below (blue) the satellite. (f-h) E-t (Energy-time) spectrogram of electron data by LEP. (i-k) E-t (Energytime) spectrogram of ion data by LEP. Detected peak energies of the downward electrons and the UFIs are also indicated over the panel (f) (downward electron) and panel (k) (upward ion).

\section{Case study of an event on 20 February 1990}

On 20 February 1990 (event 90022006), Akebono passed the nightside auroral oval at altitudes of $\sim 9900 \mathrm{~km}$ from high to low latitudes. Panels (f-k) in Fig. 3 show Energy-time (E-t) diagrams of electrons and ions. Large amounts of energetic electron precipitation at 07:23-07:40 UT are magnetospheric electrons accelerated by the parallel potential differences above the satellite. Akebono also observed UFIs (Upward Flowing Ions) at 07:23-07:40 UT, which indicate the potential difference below the satellite altitude. Therefore, Akebono was located in the middle of the parallel acceleration region during this time interval. The positive slope of the eastward magnetic field perturbation during 07:23-07:40 UT (panels a-c) corresponds to the upward field-aligned current. 


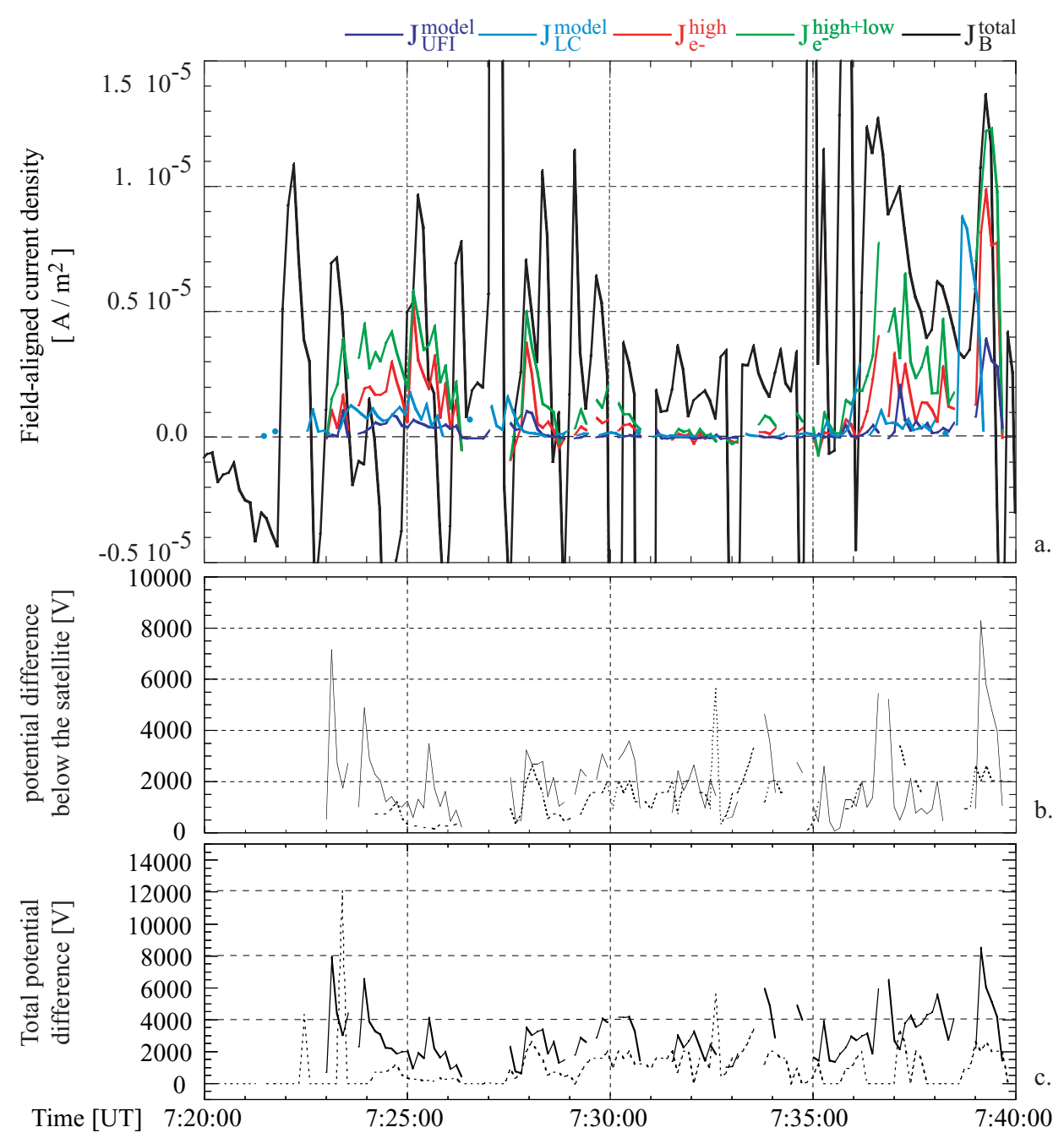

Fig. 4. (a) The field-aligned current densities estimated by the three different method (see the details in Sect. 2). $J_{B}^{\text {tot }}$ (black), $J_{e^{-}}^{\text {high }}$ (red), $J_{e^{-}}^{\text {high+low }}$ (green), $J_{\mathrm{UFI}}^{\text {model }}$ (dark blue), and $J_{L C}^{\text {model }}$ (light blue). $\phi_{b}$ calculated and $\phi_{\text {total }}$ (middle) using $\phi_{b}$ in different estimation techniques are displayed (b) and (c). Solid lines indicate the data using $\phi_{b}^{L C}$, while dashed lines indicate the data using $\phi_{b}^{\mathrm{UFI}}$.

The field-aligned current estimated from the magnetic field is also shown in panel d. In this event, the upward fieldaligned current region (enclosed area with red dash) agrees well with the energetic electron precipitation region, and this is consistent with the expectation that the field-aligned current is related to the particle acceleration. On the other hand, the field-aligned current was not simply proportional to the potential difference (black line in panel e) like Knight's relation.

Figure 4a shows the field-aligned current densities estimated in three different methods. If Knight's relation is correct, all the current densities estimated by the three different techniques should be consistent. However, the currents do not necessarily agree well. First of all, when the magnetospheric electrons are accelerated adiabatically, as assumed in Knight's theory, Knight's model current should agree with $J_{e^{-}}^{\text {high }}$. However, comparing $J_{\text {UFI }}^{\text {model }}$ (dark blue in panel a) and
$J_{e^{-}}^{\text {high }}$ (red), $J_{e^{-}}^{\text {high }}$ is much higher than the model. This may be due to the underestimation of the potential difference. Reiff et al. (1988) argued that the energy degradation of UFIs may occur owing to the two-stream instability of hydrogen and oxygen ions. In this case, $\phi_{b}^{\mathrm{UFI}}$ might be underestimated. The potential differences below the observation point estimated from UFI's peak energy ( $\phi_{b}^{\mathrm{UFI}}$, black) and electron loss cone technique ( $\phi_{b}^{L C}$, blue) are shown in panel (b). It can be noticed that $\phi_{b}^{L C}$ is often larger than $\phi_{b}^{\mathrm{UFI}}$, as is consistent with the previous results (Reiff et al., 1993; Sakanoi et al., 1995). With this effect, the value of $J_{L C}^{\text {model }}$ (blue in panel a) often reaches the value of $J_{e^{-}}^{\text {high }}$ (red). For example, $J_{e^{-}}^{\text {high }}$ and $J_{L C}^{\text {model }}$ are almost the same at 07:39 UT while $J_{\mathrm{UFI}}^{\text {model }}$ is much smaller than the others. The agreement of $J_{e^{-}}^{\text {high }}$ and $J_{\mathrm{UFI}}^{\text {model }}$ indicates that one of the assumptions in Knight's relation, that electrons with energies higher than $E_{\text {peak }}$ are adiabatically accelerated by the potential drop, is valid. 

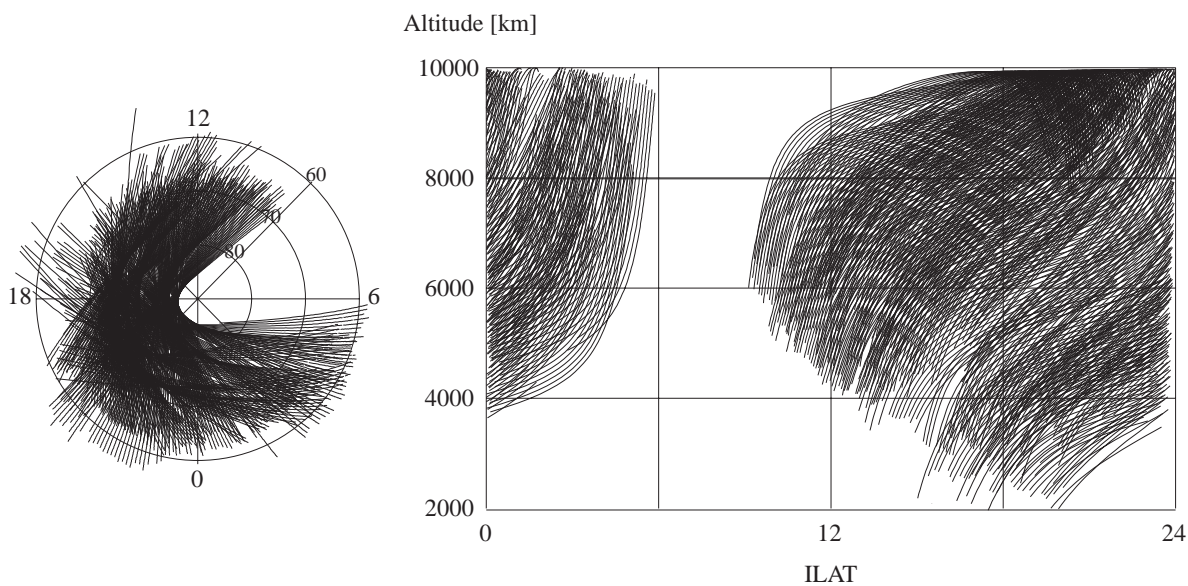

Fig. 5. Plots of the Akebono orbit during December 1989 to February 1990. For most of the time, the Akebono satellite was located at the high altitudes above nightside auroral region.
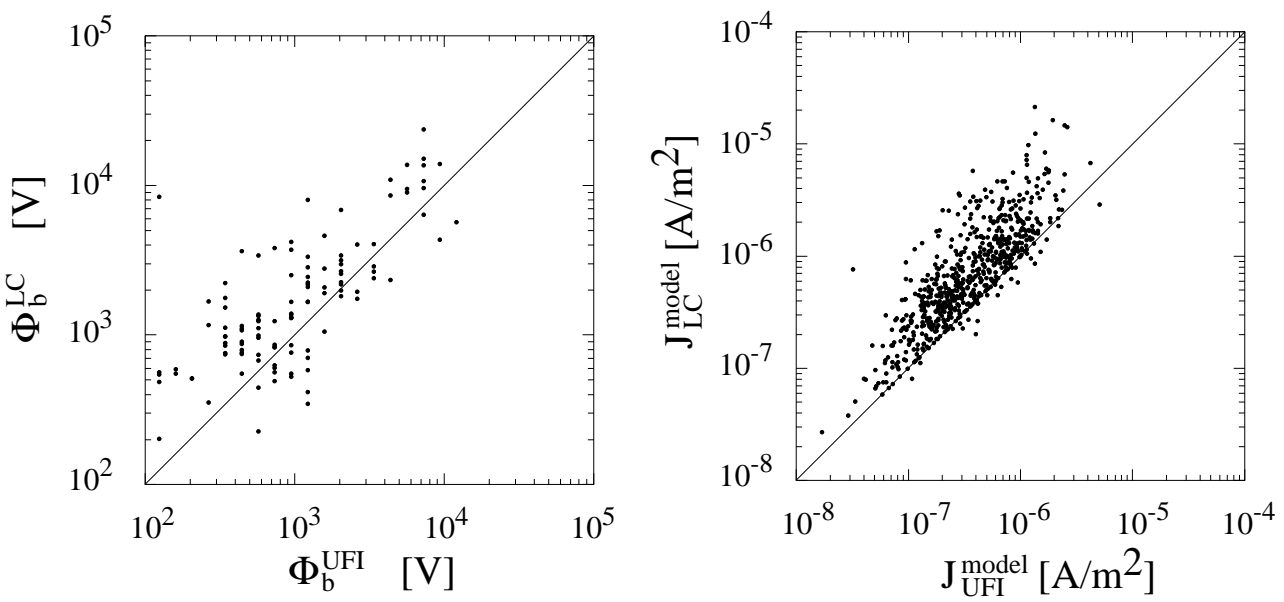

Fig. 6. Left: Comparison of $\phi_{b}$ estimated from two different techniques. Right: Comparison of $J_{L C}^{\text {model }}$ and $J_{\mathrm{UFI}}^{\text {model }}$.

On the other hand, $J_{B}^{\text {tot }}$ often significantly exceeds $J_{\mathrm{UFI}}^{\text {model }}$ and $J_{e^{-}}^{\text {high }}$. Thus, there must be other particles (besides the magnetospheric primary electrons) for charge carriers of the current. We suggest that the low energy electrons also contribute to the current. It is found, in Fig. 4a, that the values of $J_{e^{-}}^{\text {low+high }}$ (green), which is the current density carried by electrons over the whole energy range above $100 \mathrm{eV}$, are often significantly larger than $J_{e^{-}}^{\text {high }}$ and $J_{\mathrm{UFI}}^{\text {model }}$. Besides, $J_{e^{-}}^{\text {low+high }}$ often reaches $J_{B}^{\text {tot }}$, especially near the edge of the upward field-aligned current region, where the potential differences above the satellite altitudes are small (07:39 UT, for example). Hence, the electrons with energies lower than $E_{\text {peak }}$ significantly contribute to the upward field-aligned current. We have further examined the relationship between the field-aligned current and the potential difference for five more events observed in February 1990 (not shown), and obtained similar results to the event 90022006 .

\section{Statistical result at high altitudes}

In order to generalize the result in the previous section, we examined the field-aligned current in the particle acceleration region statistically, using the data during a period from November 1989 to February 1990. Figure 5 illustrates the Akebono orbits in this period. Akebono has mainly observed the nightside high altitude auroral region.

Figure 6 shows the comparison of the results using the two different techniques for potential difference. The left panel shows the differences between $\phi_{b}^{L C}$ and $\phi_{b}^{\mathrm{UFI}}$. In many cases, $\phi_{b}^{L C}$ is larger than $\phi_{b}^{\mathrm{UFI}}$. As a result, $J_{L C}^{\text {model }}$ is larger than $J_{\text {UFI }}^{\text {model }}$ in most cases, as shown in the right panel. Hereafter, we have used $\phi_{b}^{L C}$ for the calculation of the model currents in the following analysis.

Figure 7 shows the comparison between currents estimated by three different methods. In the left panel, $J_{L C}^{\text {model }}$ and $J_{e^{-}}^{\text {high }}$ are compared. Most data points are located around 

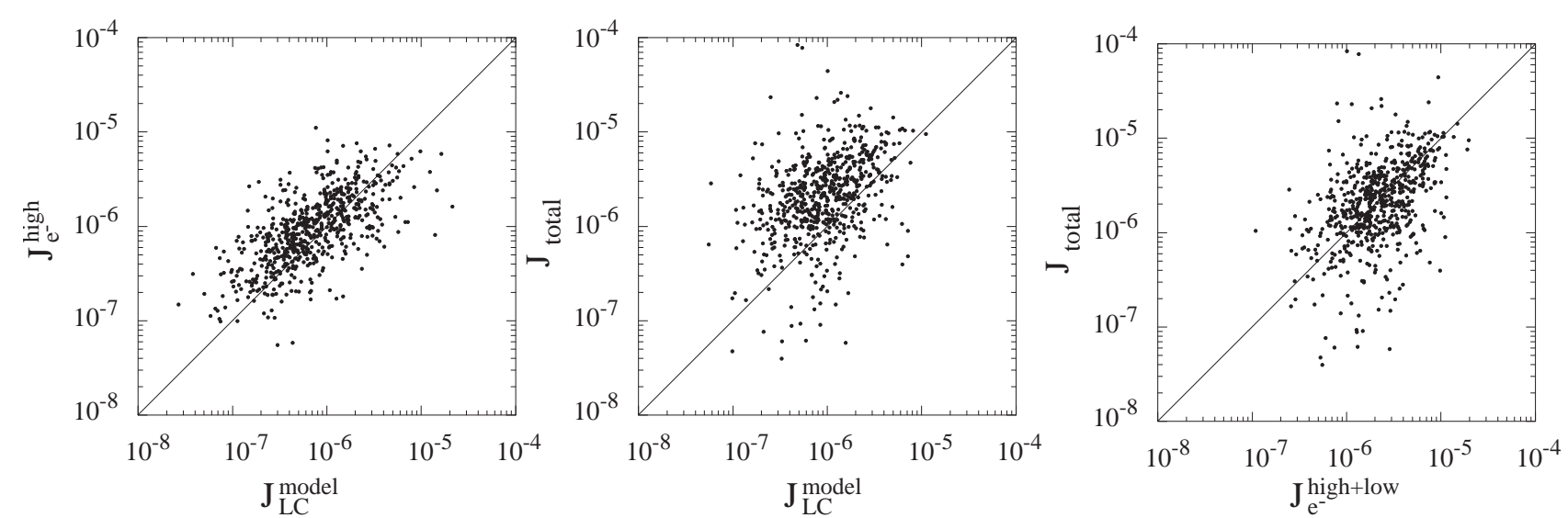

Fig. 7. Comparison of the field-aligned currents obtained during December 1989 to February 1990. Comparison between $J_{e^{-}}^{\text {high }}$ and $J_{L C}^{\text {model }}\left(\right.$ left), $J_{B}^{\text {tot }}$ and $J_{L C}^{\text {model }}$ (middle), and $J_{B}^{\text {tot }}$ and $J_{e^{-}}^{\text {low+high }}$ (right) are shown, respectively.

the line of $J_{\text {model }}=J_{e^{-}}^{\text {high }}$. Some data are still above the line of $J_{e^{-}}^{\text {high }}=J_{\text {model }}$. This may be due to the electron heating effect or non-isotropic distribution of the source electrons (Reiff et al., 1988; Shiokawa and Fukunishi, 1991). Shiokawa and Fukunishi (1991) examined the heating effect as a function of

$k T_{s}=k T_{s 0}+A e V_{\|}$,

where $k T_{s}$ and $k T_{s 0}$ are the thermal energy of magnetospheric electrons at the source region and the observation point, respectively, and $A$ is the heating ratio. They found the heating ratio of $A$ 0.05-0.4. Assuming Eq. (9), the model current can be estimated to increase by a factor of 1.5 (the details of the calculation are not shown here). Hence, the electron heating effect may account for the underestimation of the model current.

Knight's model current might be underestimated if the electrons at source region do not have an isotropic Maxwellian distribution function. It is pointed out that the magnetospheric electrons often have bi-Maxwellian distribution or Kappa distribution (e.g., Fridman and Lemair, 1980). In case when the source electron had bi-Maxwellian distribution function the model current would be modified as:

$J_{\|}=\frac{\sqrt{k T_{s} \|}}{k T_{S \perp}} \frac{e^{2} N_{S}}{\sqrt{2 \pi m_{e}}} V_{\|}$,

where $k T_{s \|}$ and $k T_{s \perp}$ are the parallel and perpendicular thermal energies of the source electrons (Fridman and Lemair (1980)). Here, the large accelerating potential $\left(e V_{\|} \ll k T_{S}\right)$ is assumed. The ratio of $k T_{s \|}$ to $k T_{s \perp}$ is typically less than 1.5.

The above electron heating or anisotropic electron effects can cause the underestimation of Knight's model current, but we can say that Knight's model predicts well the fieldaligned current densities contributed by the magnetospheric electrons. This result is consistent with the event study in 90022006. However, in the middle panel, most data points of $J_{B}^{\text {tot }}$ are much larger than $J_{L C}^{\text {model }}$. On the other hand,
$J_{e^{-}}^{\text {low+high }}$, shown in right panel, have a good agreement with $J_{B}^{\text {tot }}$. The above results are all consistent with the event study in 20022006.

Our statistical study shows that the lower-energy electrons also contribute significantly to the total field-aligned current in the particle acceleration region, which cannot be explained by the time-stationary adiabatic Knight's model. This is consistent with the previous results obtained by Sakanoi et al. (1995), that the $J_{B}^{\text {tot }}$ is significantly larger the Knight's model current $\left(J_{\text {UFI }}^{\text {model }}\right)$. Contrary to these Akebono results, there have been several reports that the field-aligned currents are well explained by Knight's model, using the satellite data observed at lower altitudes (e.g. Lyons et al., 1979; Weimer et al., 1987; Lu et al., 1991).

The discrepancy between the results obtained by these satellites and Akebono may be due to the difference in their observation altitudes.

\section{Statistical survey on altitudes, latitudes, local times, and seasons}

Since 1989, Akebono has obtained a large amount of data in the auroral particle acceleration region. We have surveyed 8165 Akebono passes over the auroral region during summer (May to July) and winter (November to January) of 1989 to 1997 . Data numbers of events, in which the energetic electrons with accelerated Maxwellian distributions have been observed, are shown in Fig. 1.

Figure 8 shows $J_{e^{-}}^{\text {low+high }}$ versus $J_{e^{-}}^{\text {high }}$ observed in the winter hemisphere at altitudes above (left) and below (right) $6000 \mathrm{~km}$, respectively. The lines of $J_{e^{-}}^{\text {low+high }}=J_{e^{-}}^{\text {high }}$ and $J_{e^{-}}^{\text {low+high }}=2 J_{e^{-}}^{\text {high }}$ are displayed in each panel. At high altitudes one can see a significantly large number of data above the line of $J_{e^{-}}^{\text {low }+ \text { high }}=2 J_{e^{-}}^{\text {high }}$, while few data exist above the line of $J_{e^{-}}^{e^{-}}=2 J_{e^{-}}^{\text {high }}$ in the case of low altitudes. This result indicates that lower-energy electrons contribute 

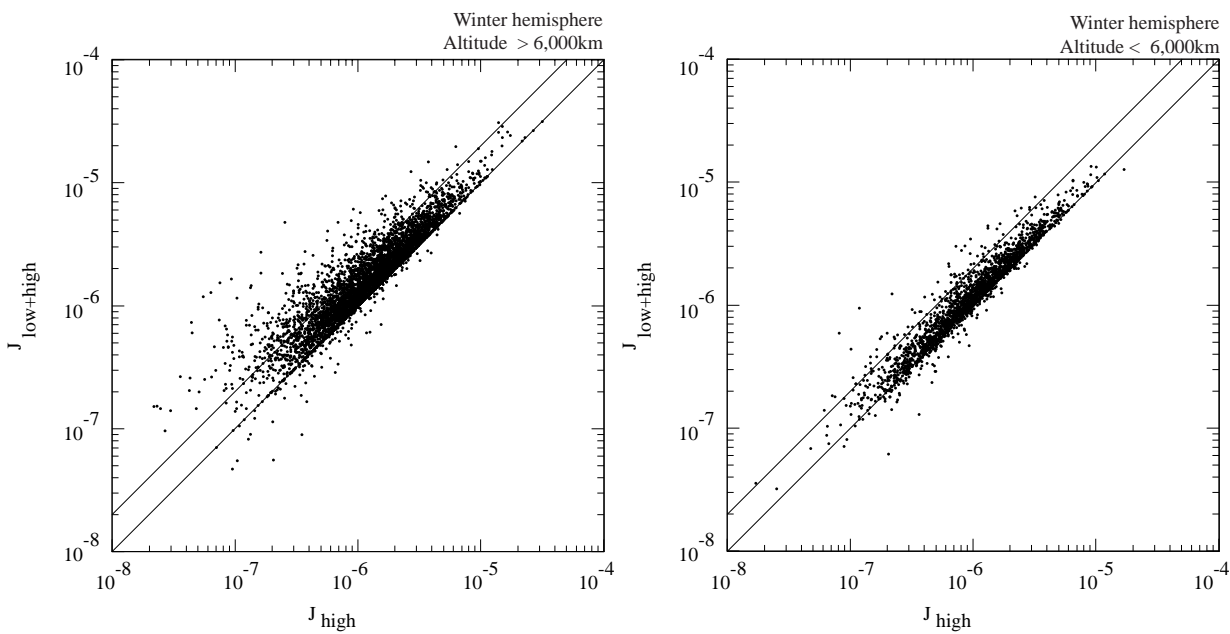

Fig. 8. The plot of $J_{e^{-}}^{\text {low+high }}$ versus $J_{e^{-}}^{\text {high }}$ obtained at high altitudes (above $6000 \mathrm{~km}$ ) and low altitudes (below $6000 \mathrm{~km}$ ), respectively. These data were obtained in the winter hemisphere. Two lines in each panel indicate the line of $J_{e^{-}}^{\text {low }+ \text { high }}=J_{e^{-}}^{\text {high }}$ and $J_{e^{-}}^{\text {low+high }}=2 J_{e^{-}}^{\text {high }}$, respectively.

to the current at higher altitudes, whereas high-energy electrons mainly carry the currents at lower altitudes. Note that during the period of November 1989 to February 1990, most of the Akebono passes were located at altitudes of 8000 to $9000 \mathrm{~km}$ in winter.

We have further examined the seasonal dependence of the low-energy electrons contribution to the current. We identified such a region where the low energy electron contributes to the field-aligned current by the ratio of $J_{e^{-}}^{\text {low+high }}$ to $J_{e^{-}}^{\text {high }}$, and obtained the occurrence frequency against the acceleration event in each altitude range and season (Fig. 9). The red color indicates that the lower-energy electrons contribute to the current in more than $15 \%$ of the total cases. The highest occurrence frequency is observed in the pre-midnight region at the highest altitudes in the winter hemisphere. On the other hand, at low altitudes below $6000 \mathrm{~km}$, the ratio of $J_{e^{-}}^{\text {low+high }} / J_{e^{-}}^{\text {high }}$ is mostly less than 2 , that is, the field-aligned current density can be explained only by the electrons with energies higher than $E_{\text {peak }}$, as expected from Knight's model. These results can explain the discrepancies between the results obtained by low (e.g. DE-2) and high (Akebono) altitude satellites. A rather high occurrence frequency region is also observed at the highest altitudes around midnight in the summer hemisphere. The occurrence frequency decreases at lower altitudes. This tendency is similar to that in the winter hemisphere, however, the altitude of highest occurrence frequency in summer (above $8000 \mathrm{~km}$ ) is higher than the altitude in winter $(6000 \mathrm{~km} \sim 8000 \mathrm{~km})$. This seasonal effect of altitude is expected due to the seasonal dependence of particle acceleration region. It has recently become known that altitudes of the auroral particle acceleration region in the darkness (winter) hemisphere are lower than those in the sunlight (summer) hemisphere. Comparing the altitude profile of the particle acceleration region obtained by the Akebono satellite (see Fig. 3 of Morooka and Mukai, 2003), the high occurrence of $J_{e^{-}}^{\text {low+high }} / J_{e^{-}}^{\text {high }}>2$ region is located in the middle of the acceleration region. Figure 10 shows the ratio of $J_{e^{-}}^{\text {low+high }} / J_{e^{-}}^{\text {high }}$ versus the ratio of potential difference $\phi_{a} / \phi_{b}$ UFI. Mean values of the current ratio as a function of potential ratio are displayed in red diamonds. The high ratio of $J_{e^{-}}^{\text {low+high }}$ to $J_{e^{-}}^{\text {high }}$ occurs especially when the ratio of the potential differences above and below the satellite altitudes are unity. This is consistent with the idea that the low energy electrons contribute to the field-aligned current in the middle of the acceleration region. This result can explain that the Akebono satellite, which has a high altitude orbit, often observed $J_{B}^{\text {tot }}$ larger than Knight's model current, while other low altitude satellites obtained $J_{B}^{\text {tot }}$ consistent with Knight's relation.

\section{Discussion}

Using the magnetic field and particle data obtained by the Akebono satellite, the current-voltage relationship in the auroral particle acceleration region has been statistically examined. The obtained results are summarized as follows:

1. The upward field-aligned current, $J_{B}^{\text {tot }}$, was often several times larger than Knight's model current, $J_{\mathrm{UFI}}^{\text {model }}$ and $J_{L C}^{\text {model }}$. item $J_{e^{-}}^{\text {high }} \sim e q J_{\text {model }}$, that is, the electron with energies higher than $E_{\text {peak }}$ is accelerated adiabatically, which is consistent with Knight's assumption. On the other hand, $J_{B}^{\text {tot }} \sim e q J_{e^{-}}^{\text {low+high }}$, which indicates that the electrons with energies lower than $E_{\text {peak }}$ significantly contribute to the upward field-aligned current.

2. Such low-energy electrons contribute to the current, especially in the middle of the particle acceleration region, 


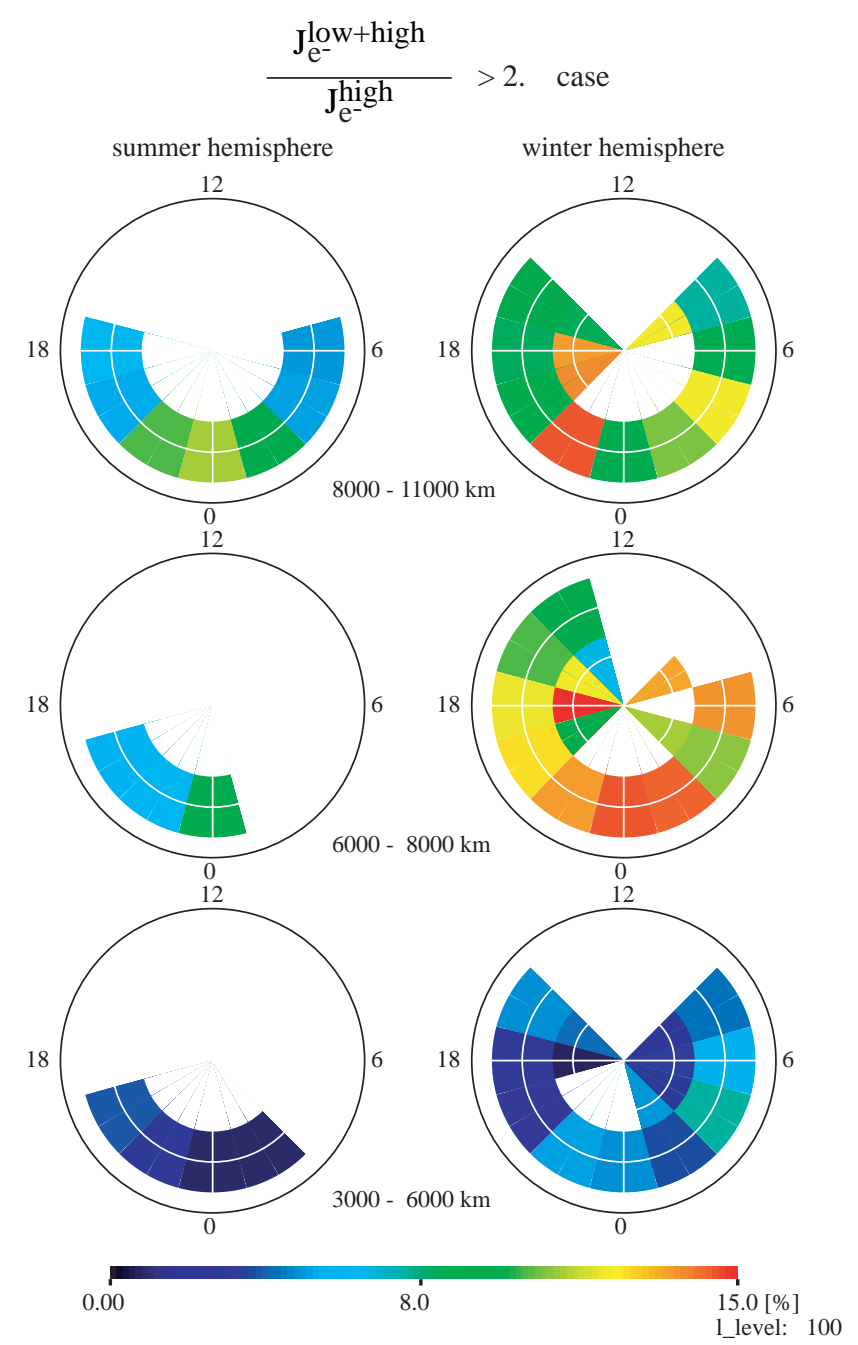

Fig. 9. The occurrence frequency of the case $\frac{J_{e^{-}}^{\text {low+high }}}{J_{e^{-}}^{\text {high }}}>2.0$ in the particle acceleration region. The color bar shows the linear definition of the occurrence with maximum value as $15 \%$.

where the parallel electric fields exist above and below the observation point. The altitude of the region depends on season in the same way as the seasonal dependence of the particle acceleration region.

The above results are not inconsistent with previous works that the upward field-aligned current can be explained by Knight's model at low altitudes (see Fig. 8). Furthermore, even at high altitudes, the result of $J_{e^{-}}^{\text {high }} \sim J_{L C}^{\text {model }}$ suggests that Knight's model predicts well the current carried by the electrons with energies higher than $E_{\text {peak }}$, though $J_{e^{-}}^{\text {high }}$ tends to be larger (but with a factor of 2) than $J_{L C}^{\text {model }}$. More surprising and the most important question is why and how such low-energy electrons dominantly contribute to the current. In the adiabatic model, such as described by Chiu and Schulz (1978) and Knight (1973), the low-energy electrons are considered as secondary electrons originating from

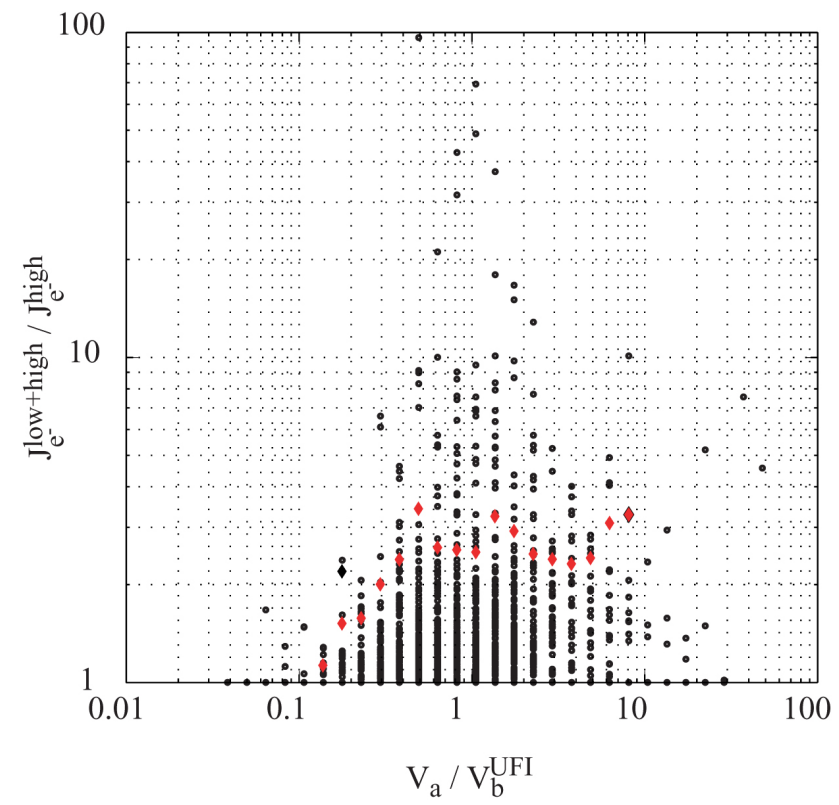

Fig. 10. Scatter plot of the ratio of $J_{e^{-}}^{\text {low+high }}$ to $J_{e^{-}}^{\text {high }}$ and the ratio of the potential difference above and below the observation point. Red diamonds indicate the mean values of the current ratio $\left(J_{e^{-}}^{\text {low }+ \text { high }} / J_{e^{-}}^{\text {high }}\right)$ as a function of potential ratio $\left(\phi_{a} / \phi_{b}^{\mathrm{UFI}}\right)$.

the ionosphere or trapped ones between the magnetic mirror point and the particle acceleration region above the satellite altitude. The trapped region of electron phase space is considered as a "forbidden" region where the particles from either side of the acceleration region cannot enter adiabatically, and therefore, they cannot contribute to the current in the adiabatic model. However, in the middle of the auroral particle acceleration region, we often observed a significant amount of electrons in the "forbidden" region of phase space, and they contribute to the current. We will discuss what the origin of the low-energy electrons is and how they contribute to the current.

One candidate for the low-energy electron population is the ionospheric electron. Sakanoi et al. (1995) have proposed that the upgoing electrons, which were the carriers of the downward field-aligned current, may be transported into the acceleration region and become a new carrier of the upward current. In order to transport the cold electrons into the inverted-V region, an $\boldsymbol{E} \times \boldsymbol{B}$ drift caused by the convection electric field along the current sheet may help. However, an $\boldsymbol{E} \times \boldsymbol{B}$ drift force can supply cold electrons only in a very narrow edge region of inverted- $\mathrm{V}$, since the velocity of electrons are high and they can pass through the acceleration region quickly. Lotko (1996) suggested that the acceleration region consists of many double layers and the convection electric field can make the collimated electron spectra near the edge of the inverted- $\mathrm{V}$ region. The pitch angle scattering of the cold electrons is also assumed in this model. Their model can explain the presence of such low energy electrons in about $10 \mathrm{~km}$ width at the edge of the inverted- $\mathrm{V}$ region. However, 
EXOS-D 90022006 07:35:48

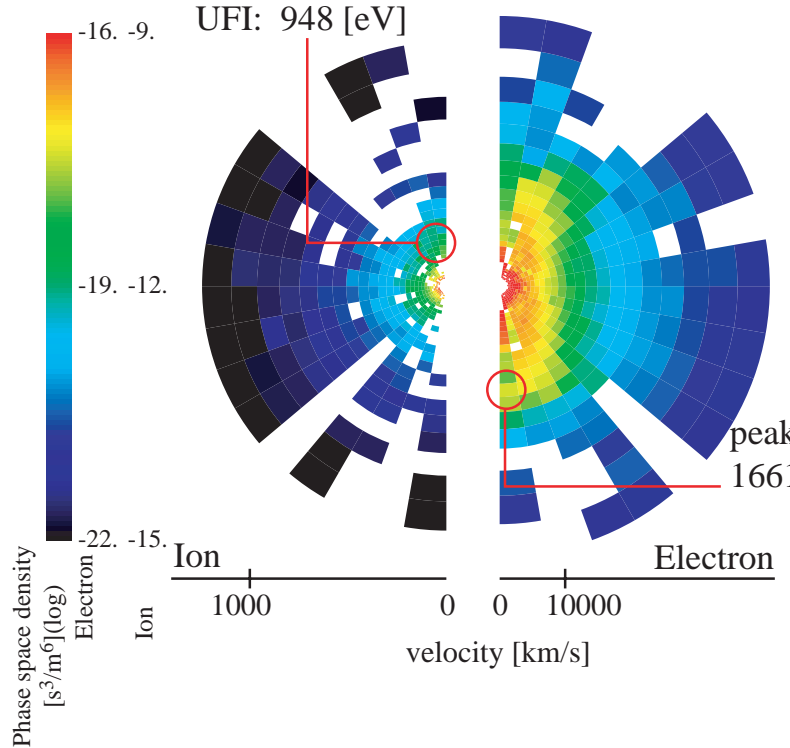

a

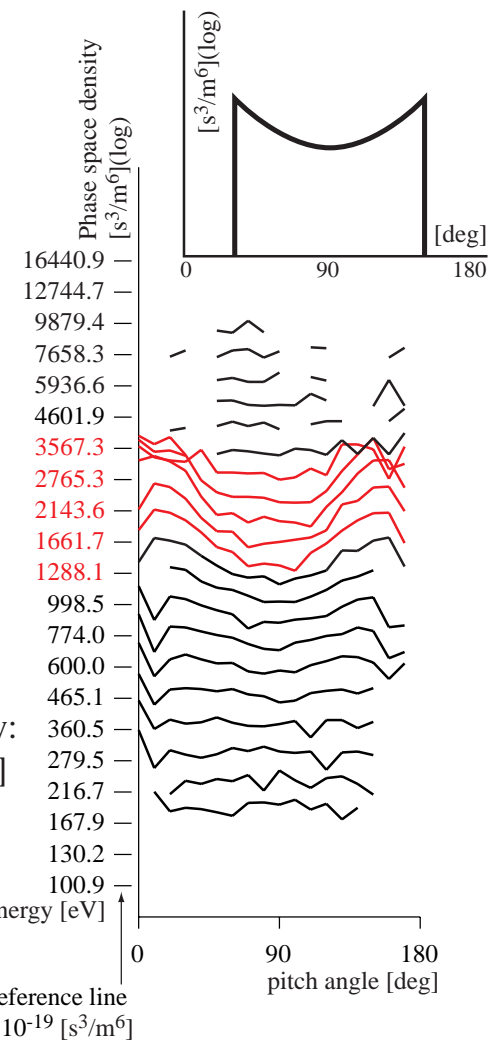

b

Fig. 11. Distribution of electrons and ions observed by Akebono. (a) Distribution function of electrons and ions, respectively, in the velocity space. The electron's distribution function shows a "cylindrical" distribution. (b) Up: Schematic model pitch angle distribution function by Eq. (11). Bottom: Pitch angle distributions of electrons at different energies.

it is also difficult to explain the existence of the low-energy electrons deep inside the acceleration region. Wave-particle interaction, such as pitch angle scattering by auroral kilometric radiation (AKR), suggested by Calvert (1982), Calvert (1987), can also transport cold electrons to the acceleration region. However, in this case, the assumption of adiabatic acceleration is violated, which is inconsistent with the result that Knight's model current is well predicted.

So far, we have discussed the possible mechanisms to let the low energy electrons contribute to the current on the time-stationary condition, however, it is concluded that the ionospheric electrons cannot contribute to the current significantly in the time-stationary model. In order to examine the origin of the low-energy electrons, we have examined the electron distribution functions in more detail, and found that the distribution function of the trapped electrons has some important characteristics, which cannot be simply explained by the conventional adiabatic model. We will show the electron distribution functions which have been obtained in the upward field-aligned current region, as follows.

Figure 11 shows the electron distribution function observed in the event 90022006 . In this example, the trapped electron distribution function is elongated along the magnetic field. This pattern is often observed in the middle of the upward field-aligned current region. Machida (personal communication) has also found similar electron distributions to the one in Fig. 11 in the Akebono data, and called it a "cylindrical" distribution. Using Viking data, Louarn et al. (1991) also found similar electron distributions, and suggested that it could be explained if one considers the time growth of the parallel potential differences above the spacecraft. The idea of the growing potential differences was first proposed by Eliasson et al. (1979). In general, the time that the electrons go to the magnetic mirror point and back again is so fast that the electric fields can be considered as static. However, when the potential difference above the observation point increases from 0 to $\phi$ with time comparable to the electron bounce motion, mirroring electrons will be reflected back by the increased potential difference, and will be trapped between the acceleration region and the magnetic mirror point. Eliasson et al. (1979) predicted the pitch angle distribution of such trapped electrons from Liouville's theorem as follows:

$f(E, \alpha)=N_{S}\left(\frac{m_{e}}{2 \pi k T_{S}}\right)^{\frac{3}{2}} \exp \left\{-\frac{E}{k T_{S}} \frac{B_{S}}{B_{o}} \sin ^{2} \alpha\right\}$,

where $E$ is the electron energy, $\alpha$ is the pitch angle, and $B_{s}$ and $B_{o}$ are the magnetic intensities at the source and observation regions, respectively. Details of the pitch angle 


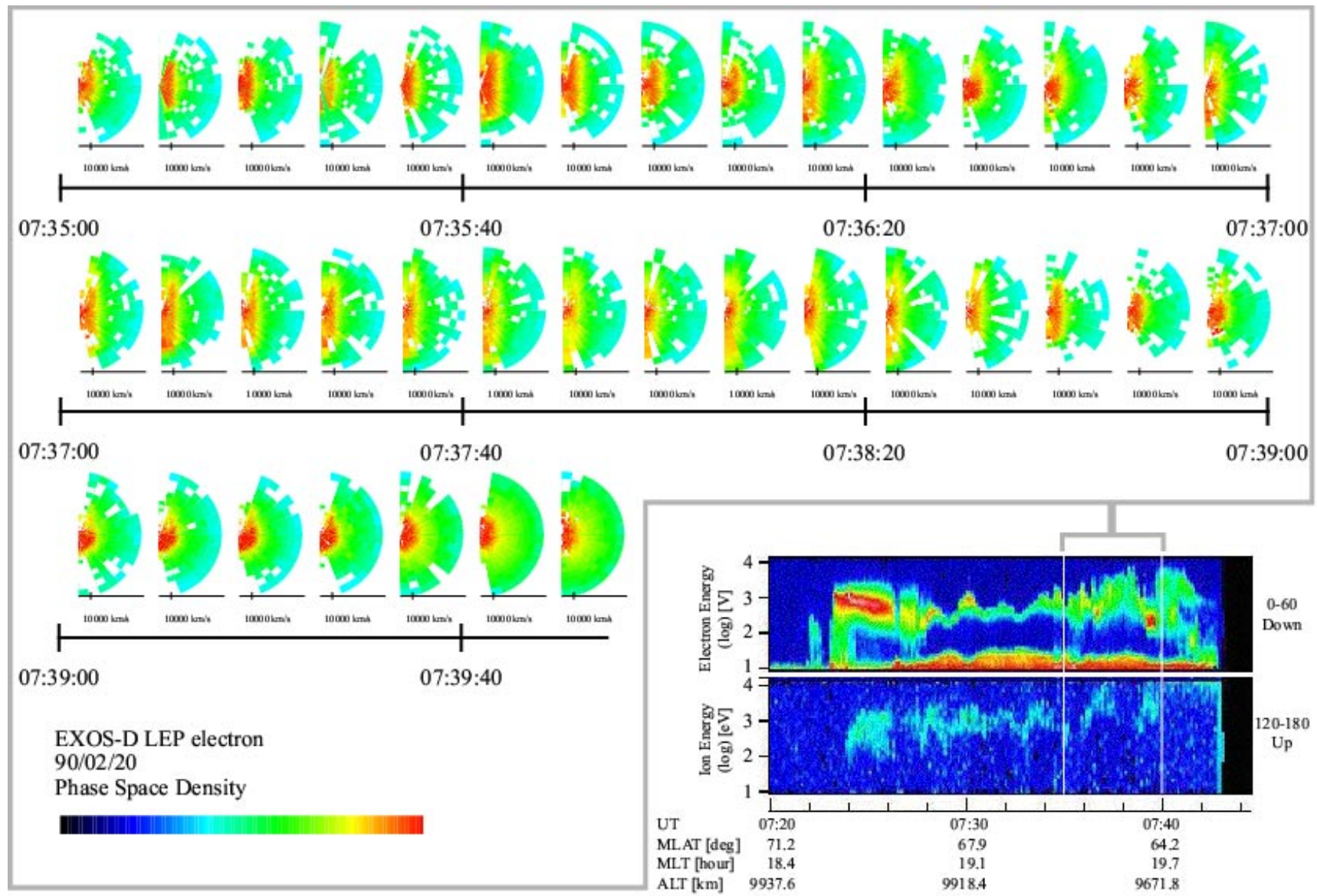

Fig. 12. The pitch angle distributions functions in the phase space obtained during 07:25 UT-07:40 UT in event 90022006 . The cylindrical distributions are observed for a long time. The electrons with energies lower than $E_{\text {peak }}$ have contributed to the field-aligned currents during this interval.

distribution functions of electrons are shown in panel (b). Note that the cylindrical distribution has a similar sine curved pitch angle distribution to those expected by Eq. (11), as shown in upper part of panel (b). Figure 12 shows a time series of the electron distributions obtained in the event 90022006, in which the cylindrical electron distribution is observed for a significantly long period, from 07:25 UT to 07:40 UT. This fact implies that the potential differences above the observation region varied in time. Note, however, that the symmetrically trapped electrons does not significantly contribute to the current.

Another peculiar feature, as shown in Fig. 13, has been observed near the edge of the upward field-aligned current region, where the low-energy electrons significantly contribute to the upward current. This distribution contains several important characteristics. First, there is a significant amount of downward electrons in the forbidden region (at the energies below $\left.E_{\text {peak }}\right)$. Second, the electron's loss cone angle was extremely large. In this case, the potential difference below the observation point, estimated from the UFI's peak energy, was about $2040 \mathrm{eV}$. On the other hand, the potential difference below the satellite estimated from the electron's loss cone was about $4840 \mathrm{eV}$, which is more than twice the value estimated by the UFI peak energy. Reiff et al. (1988) have also suggested that the potential difference estimated by the UFI peak energy is often smaller than that estimated from electron loss cone because of thermalization during the transport. However, the present 2.4-times difference is too large to be explained only by the thermalization process (Reiff et al., 1988, showed only $30 \%$ difference). The time variability of the acceleration region is also one possible mechanism to explain the energy difference, and it can also explain the large difference between the UFI peak energy and the potential difference estimated from the electron loss cone.

If the potential difference well below the observation point varies rapidly, it takes a long time for ions to reach the observation point, while electrons can respond quickly to the time variability. In this case, the trapped electrons can also contribute to the field-aligned currents.

The distribution function of Fig. 13 has another important characteristic, that is, the presence of the upward electrons with energies higher than the peak energies of downward electrons. $E_{\text {peak }}$ of the upward electrons with the pitch angle of $160^{\circ}$ was $1288 \mathrm{eV}$, while $E_{\text {peak }}$ of the downward (in $0^{\circ}$ ) 


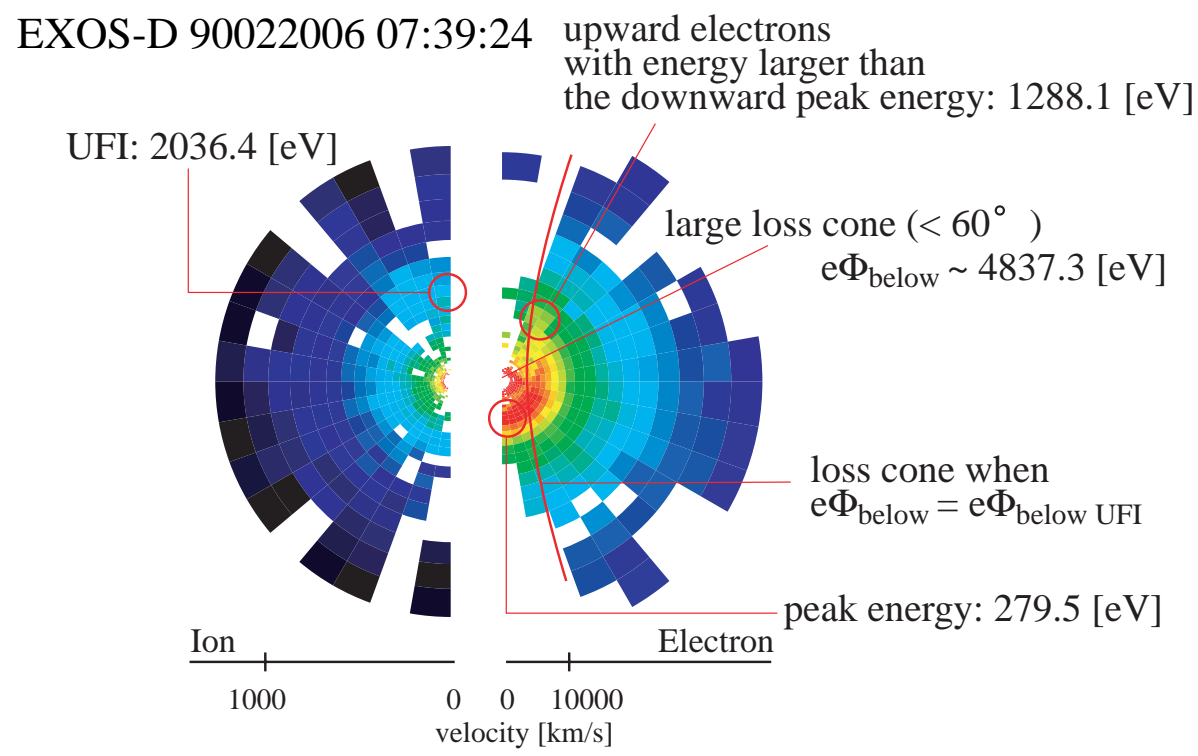

Fig. 13. Distribution functions of electrons and ions observed by Akebono. The format is the same as panel (a) of Fig. 11. The electron loss cone is extremely large, which indicates a potential difference much larger than that estimated by the UFI's peak energy. Upgoing high-energy electrons with larger energy than that of the downward peak energy are also observed.

electrons was only $279 \mathrm{eV}$. This can be explained by a rapid decreasing of the potential difference below the observation point, which also indicates the time variability of the acceleration region.

We expect that the following scenario is possible to explain the mechanism to make the low-energy electrons contribute to the current. First, the potential difference increases slowly (in order of several s) above the observation point, which can make the population of the electrons in the "forbidden" region of phase space. After that, a rapid increase of the potential difference below the observation point is necessary to make a large loss cone and allow the low-energy electrons to contribute to the field-aligned current.

The above features in electron distribution functions indicate that the particle acceleration region often varies in time, which is inconsistent with the time-stationary assumption of Knight's model. We emphasize that the low-energy electrons, which contribute to the currents in such a timevariable case, exist in the "forbidden" region of phase space in the time-stationary model. When we consider the timevariability of the acceleration region, the ionospheric cold electron, which successfully moves into the inverted-V region from the edge region, can be a candidate for the lowenergy electrons which contribute to the current. However, the pitch angle of such electrons will probably be very small, which is inconsistent with our result that a part of the fieldaligned current is carried by the electrons in the "forbidden" region. In addition, they can only explain the low-energy electrons at a narrow edge area of the acceleration region, as mentioned above. Therefore, the ionospheric electrons cannot be a charge carrier of the filed-aligned current.
As suggested by Eliasson et al. (1979), the electron population in the "forbidden" region of the phase space can be explained by the magnetospheric electrons in a time varying acceleration region. The characteristics of the electron distribution function in Fig. 13 indicate that the potential difference below the observation point increases and decreases, repetitively. Therefore, a combination of the distribution functions in Figs. 11 and 13 implies that the particle acceleration region around the Akebono satellite altitude varies in time, and it makes it possible for the electrons in the "forbidden" region of phase space originating from the magnetosphere to contribute to the field-aligned current. A similar electron distribution function to Fig. 13 has been observed above the auroral region by the Viking satellite (André and Eliasson, 1992), and the fluctuating acceleration region below the spacecraft has been suggested to explain the feature. Their model seems to be consistent with our consideration. In the time-constant Knight's model, many parts of the precipitating electrons from the magnetosphere are reflected back by the magnetic mirror force. However, in the time-varying acceleration region case in this study, more magnetospheric electrons may precipitate into the ionosphere.

Finally, secondary electrons are also a candidate for the current carrier, when we consider the time variability of the acceleration region. As investigated by Pulliam et al. (1981), a significant amount of secondary electrons could be supplied by iterating the scattering and reflection above the auroral region. However, their population is mostly generated at low altitudes below the acceleration region and they have difficulty in reaching the altitude of the acceleration region, where the Akebono satellite is located. 


\section{Conclusions}

On the basis of the LEP (low energy particle) and MGF (magnetometer) data obtained by Akebono, we have statistically examined the current-voltage relationship in the particle acceleration region quantitatively. The particle acceleration region has generally coincided with that of the upward field-aligned current, and this fact indicats that the upward field-aligned current is related to the generation mechanism of the parallel electric field. However, the field-aligned current density was not proportional to the potential difference, on the contrary to the expectation from Knight (1973).

We have statistically examined the upward field-aligned current in terms of the current carrier, and obtained the following results: (1) The field-aligned current calculated by integrating the electron distribution above the peak energy $\left(E_{\text {peak }}\right)$ agrees well with the model current. (2) However, the real current densities estimated from the magnetometer were often several times larger than the model currents, that is, because the low-energy electrons, which exist in the "forbidden" region of phase space, often contribute to the current. (3) The low-energy electrons contribute to the current in the middle of the particle acceleration region, where the parallel potential differences exist above and below the observation point. Moreover, the existence altitude of such regions depend on season.

It is also noted that the above results are not inconsistent with previous works that upward field-aligned current can explain Knight's model at low altitudes. From the altitude dependence of the charge carrier of the current (Fig. 8), we conclude that the discrepancy between the results obtained by the low-altitudes satellites and Akebono is due to the difference in their observation altitudes.

We have examined the electron distribution functions in detail, and suggest that the low-energy electrons contribute to the field-aligned current when the particle acceleration region varies rapidly in time. The electron distribution functions showed some important characteristics which indicate the time variability of the acceleration region around the observation altitudes. By considering the time-variability of the acceleration region, the magnetospheric electron population may precipitate into the ionosphere more than expected by the time-stationary model.

Acknowledgements. The authors would like to express her acknowledge to members of the Akebono science team. We are indebted to M. André for his valuable suggestions and careful reading of this manuscript. The authors would also like to thank P. Janhunen and A. Olsson for much valuable advice and comment.

Topical Editor T. Pulkkinen thanks T. Sakanoi and another referee for their help in evaluating this paper.

\section{References}

André, M. and Eliasson, L.: Electron acceleration by low-frequency electric field fluctuations: Electron conics, Geophys. Res. Lett., 19, 1073-1076, 1992.

Borovsky, J. E.: Auroral Arc Thickness as Predicted by Various Theories, J. Geophys. Res., 98, 6101-6138, 1993.

Bryant, D. A., Hall, D. S., and Lepine, D. R.: Electron acceleration in an array of auroral arcs, Planet. Space Sci., 26, 81-92, 1978.

Bruning, K., Block, L. P., Marklund, G. T., Eliasson, L., Pottelette, R., Murphree, J. S., Potemra, T. A., and Perraut, S.: Viking observations above a postnoon aurora, J. Geophys. Res., 95, 60936049, 1990.

Calvert, W.: A feedback model for the source of auroral kilometric radiation, J. Geophys. Res., 87, 8199-8214, 1982.

Calvert, W.: Auroral precipitation caused by auroral kilometric radiation, J. Geophys. Res., 92, 8792-8794, 1987.

Chiu, Y. T. and Schulz, M.: Self-consistent particle and parallel electrostatic field distributions in the magnetosphericionospheric auroral region, J. Geophys. Res., 83, 629-642, 1978.

Cladis, J. B. and Sharp, R. D.: Scale of electric field along magnetic field in an Inverted V event, J. Geophys. Res., 84, 6564-6572, 1979.

Eliasson, L., Holmgren, L., and Rönnmark, K.: Pitch angle and energy distributions of auroral electrons measured by the ESRO 4 satellite, Planet. Space Sci., 27, 87-97, 1979.

Elphic, R. C., Bonnell, J. W., Strangeway, R. J., Kepko, L., Ergun, R. E., McFadden, J. P., Carlson, C. W., Peria, W., Cattell, C. A., Klumpar, D., Shelley, E., Peterson, W., Moebius, E., Kistler, L., and Pfaff, R.: The auroral current circuit and field-aligned currents observed by FAST, Geophys. Res. Lett., 25, 2033-2036, 1998.

Frank, L. A. and Ackerson, K. L.: Observations of charged particle precipitation into the auroral zones, J. Geophys. Res., 76, 36123643, 1971.

Frey, H. U., Haerendel, G., Clemmons, J. H., Boehm, M. H., Vogt, J., Bauer, O. H., Wallis, D. D., Blomber, L., and Lühr, H.: Freja and ground-based analysis of inverted-V events, J. Geophys. Res., 103, 4303-4314, 1998.

Fridman, M. and Lemair, J.: Relationship between auroral electron fluxes and field aligned electric potential differences, J. Geophys. Res., 85, 664-670, 1980.

Fukunishi, H., Fujii, R., Kokubun, S., Hayashi, K., Tohyama, T., Tonegawa, Y., Okano, S., Sugiura, M., Yumoto, K., Aoyama, I., Sakurai, T., Sato, T., Iijima, T., Nishida, A., and Natori, M.: Magnetic field observations on the Akebono (EXOS-D) satellite, J. Geomagn. Geoelectr., 42, 385-409, 1990.

Gurgiolo, C. and Burch, J. L.: Simulation of electron distributions within auroral acceleration regions, J. Geophys. Res., 93, 39894003, 1988.

Haerendel, G., Frey, H. U., Bauer, O. H., Rieger, E., Clemons, J., Boehm, M. H., Wallis, D. D., and Lühr, H.: Inverted-V events simultaneously observed with the Freja satellite and from the ground, Geophys. Res. Lett., 21, 1891-1894, 1994.

Kaya, N., Matsumoto, H., and Yamagish, H.: Rocket measurement of auroral keV electron fluxes in Antarctica, Mem. Matl. Inst. Polar Res., 18, 427, 1981.

Knight, S.: Parallel electric fields, Planet. Space Sci., 21, 741-750, 1973.

Lotko, W.: Diffusive acceleration of auroral primaries, J. Geophys. Res., 91, 191-203, 1996. 
Louarn, P., Quéau, D. L., and Roux, A.: Formation of electron trapped population and conics inside and near auroral acceleration regions, Ann. Geophys., 9, 553-563, 1991.

Lu, G., Reiff, P. H., Burch, J. L., and Winningham, J. D.: On the auroral current-voltage relationship, J. Geophys. Res., 96, 3523$3531,1991$.

Lyons, L.: Generation of large-scale regions of auroral currents, electric potentials, and the Precipitation by the Divergence of the convection electric field, J. Geophys. Res., 85, 17-24, 1980.

Lyons, L., Evans, D. S., and Lundin, R.: An observed relation between magnetic field aligned electric fields and downward electron energy fluxes in the vicinity of auroral forms, J. Geophys. Res., 84, 457-467, 1979.

Morooka, M. and Mukai, T.: Density as a controlling factor for seasonal and altitudinal variations of the auroral particle acceleration region, J. Geophys, Res., 108, 1306, doi:10.1029/2002JA009786, 2003.

Mukai, T., Kaya, N., Sagawa, E., Hirahara, M., Miyake, W., Obara, T., Miyaoka, H., Machida, S., Yamagishi, H., Ejiri, M., Matsumoto, H., and Itoh, T.: Low energy charged particle observations in the "Auroral" magnetosphere: results from the Akebono (EXOS-D) satellite, J. Geomagn. Geoelectr., 42, 479-496, 1990.

Olsson, A., Andersson, L., Eriksson, A. I., Clemmonds, J., Erlandsson, R. E., Reeves, G., Hughes, T., and Murphee, J. S.: Freja studies of the current-voltage relation in the substorm related events, J. Geophys. Res., 103, 4285-4301, 1998.

Pulliam, D. M., Anderson, H. R., Stamnes, K., and Rees, M. H.: Auroral electron acceleration and atmospheric interactions: (1) rocket-borne observations and (2) scattering calculations, J. Geophys. Res., 86, 2397-2404, 1981.
Reiff, P. H., Collin, H. L., Cravan, J. D., Burch, J. L., Winningham, J. D., Shelley, E. G., Frank, L. A., and Friedman, M. A.: Determination of auroral electric potentials using high- and lowaltitude particle distributions, J. Geophys. Res., 93, 7441-7465, 1988.

Reiff, P. H., Lu, G., Barch, J. L., Winningham, J. D., Frank, L. A., Craven, J. D., Peterson, W. K., and Heelis, R. A.: On the highand low-altitude limits of the auroral electric field region, in: Physics of Auroral Plasma Dynamics, Geophys. Monogr. Ser. vol. 80, edited by: Lysak, R. L., 143-154, AGU, Washington, D.C., 1993.

Rönnmark, K.: Auroral current-voltage relation, J. Geophys. Res., 107, 1430, doi:10.1029/2002JA009294, 2002.

Sakanoi, T., Fukunishi, H., and Mukai, T.: Relationship between field-aligned currents and inverted-V parallel potential drops observed at mid altitudes, J. Geophys. Res., 100, 19343-19360, 1995.

Shiokawa, K. and Fukunishi, H.: Global characteristics of fieldaligned acceleration process with auroral arc, J. Geomag. Geoelectr., 43, 691-719, 1991.

Shiokawa, K., Fukunishi, H., Yamagishi, H., Miyaoka, H., Fujii, R., and Tohyama, F.: Rocket observation of the magnetosphereionosphere coupling processes in quiet and active arcs, J. Geophys. Res., 95, 10 679-10 686, 1990.

Weimer, D. R., Gurnet, D. A., Goertz, C. K., Menietti, J. D., Burch, J. L., and Sugiura, M.: The current-voltage relationship in auroral current sheet, J. Geophys. Res., 92, 187-194, 1987. 